\title{
Incremental Value of an Insertable Cardiac Monitor in Patients with Hypertrophic Cardiomyopathy with Low or Intermediate Risk for Sudden Cardiac Death
}

\author{
Rafi Sakhi Roy Huurman Dominic A.M.J. Theuns Arend F.L. Schinkel \\ Amira Assaf Tamas Szili-Torok Jolien W. Roos-Hesselink Michelle Michels \\ Sing-Chien Yap \\ Department of Cardiology, Erasmus MC, University Medical Center Rotterdam, Rotterdam, The Netherlands
}

\section{Keywords}

Atrial fibrillation · Hypertrophic cardiomyopathy ·

Implantable loop recorder · Insertable cardiac monitor ·

Sudden cardiac death - Ventricular arrhythmias

\begin{abstract}
Aims: The aim of the present study was to compare the rate of actionable arrhythmic events between patients with hypertrophic cardiomyopathy (HCM) who are monitored with an insertable cardiac monitor (ICM) or Holter monitoring. Methods: We studied 50 patients (mean age 52 years, $72 \%$ men) with HCM at low or intermediate risk for sudden cardiac death (SCD), of whom 25 patients received an ICM between November 2014 and February 2019. We retrospectively identified a control group of 25 patients who were matched on age, sex, and HCM Risk-SCD score category. The mean HCM Risk-SCD score was $3.41 \pm 1.31$ and $3.31 \pm 1.43$ for the ICM and Holter groups, respectively. The primary endpoint was an actionable event which was defined as an arrhythmic event resulting in a change in patient management. The secondary endpoint was the occurrence of ventricular tachycardia (VT). Results: The cumulative action-
\end{abstract}

$\begin{array}{ll}\text { karger@karger.com } & \begin{array}{l}\text { (C) } 2021 \text { The Author(s) } \\ \text { Published by S. Karger AG, Basel }\end{array} \\ \text { Www.karger.com/crd } & \begin{array}{l}\text { This is an Open Access article licensed under the Creative Commons } \\ \text { Attribution-NonCommercial-4.0 International License (CC BY-NC) } \\ \text { (http://www.karger.com/Services/OpenAccessLicense), applicable to } \\ \text { the online version of the article only. Usage and distribution for com- } \\ \text { mercial purposes requires written permission. }\end{array}\end{array}$

able event rate at 30 months was higher in the ICM group (51 vs. $27 \%$, log-rank $p$ value $<0.01$ ). De novo atrial fibrillation requiring oral anticoagulation occurred only in the ICM group $(n=3)$. Overall, 4 implantable cardioverter-defibrillators were implanted for primary prevention $(n=2$ in each group). The cumulative rate of VT episodes at 30 months was similar between groups (23\% [ICM group] vs. $42 \%$ [Holter group], log-rank $p$ value $=0.71$ ). Furthermore, the characteristics of VT were similar between groups with regard to the number of beats and rate. Conclusions: In adults with HCM, an ICM will detect more arrhythmic events requiring an intervention than a conventional Holter strategy. In contrast, the diagnostic yield of detecting VT seems similar for both groups.

(c) 2021 The Author(s)

Published by S. Karger AG, Basel

\section{Introduction}

Insertable cardiac monitors (ICMs) provide continuous rhythm monitoring and are useful for the detection of infrequent arrhythmias, especially in patients with recurrent unexplained syncope [1]. The exact role of ICMs 
in patients with hypertrophic cardiomyopathy (HCM) is less clear. The current ESC guidelines recommend that HCM patients with recurrent episodes of unexplained syncope, who are at low risk of sudden cardiac death (SCD), should be considered for an ICM $[1,2]$. Furthermore, an ICM may be considered for HCM patients with frequent unexplained palpitations [2]. However, these recommendations are based on scarce data and there are no comparative data with ambulatory Holter monitoring [3-5]. The 2014 ESC HCM guidelines recommend the use of ambulatory Holter monitoring to detect atrial and ventricular arrhythmias every 12-24 months or more often in the case of symptoms or left atrial dilatation [2]. Theoretically, the diagnostic yield for the detection of arrhythmias is higher for an ICM in comparison to intermittent Holter monitoring. This higher diagnostic yield may be clinically relevant in this patient population. For example, the detection of ventricular tachycardia (VT) may have an impact on risk stratification for SCD and the decision to implant an implantable cardioverter-defibrillator (ICD) $[2,6,7]$. Furthermore, HCM patients with documented atrial fibrillation (AF) should receive oral anticoagulation to prevent stroke [2]. In the past 5 years, we adopted a strategy to use an ICM in HCM patients at low to intermediate risk of SCD for the detection of subclinical arrhythmias, with a particular emphasis on the detection of VT. The aim of the present study was to evaluate the incremental value of ICMs compared to a conventional strategy (i.e., Holter monitoring) in adults with HCM and a low or intermediate HCM Risk-SCD score.

\section{Methods}

\section{Study Population}

This was a prospective observational study which included all consecutive adults with HCM who received a Reveal LINQ (Medtronic Inc., Minneapolis, MN, USA) between November 2014 and February 2019. All patients had an HCM Risk-SCD score $<6 \%$. The reason for an ICM was a combination of symptoms (e.g., recurrent [near] syncope, palpitations), presence of myocardial fibrosis (determined by the presence of late gadolinium enhancement [LGE] on cardiac MRI [CMR]) and/or an intermediate risk for SCD (5-year risk of SCD $\geq 4$ to $<6 \%$ ). The decision to implant an ICM was made during a Heart Team consisting of a cardiac electrophysiologist and a cardiologist specialized in HCM.

The control group was retrospectively identified from our prospective HCM registry and consisted of a matched cohort who received conventional follow-up (intermittent Holter monitoring every 6-24 months based on treating physician's discretion). Matching was based on age (with a margin of 5 years), sex, and HCM Risk-SCD score category $(<4 \%$ or $\geq 4$ to $<6 \%)$. Patients in the control group required a minimum of 1 Holter study during follow-up and at least a clinical follow-up of 1 year. This study was approved by the Ethics Committee of the Erasmus MC.

\section{ICM Settings}

All ICMs were implanted subcutaneously as recommended by the manufacturer using the incision and insertion tool. Furthermore, all patients received a handheld activator to indicate their symptoms when necessary. The ICM was routinely programmed with the following settings: tachycardia detection was set to 176 bpm for 16 beats; bradycardia setting to $30 \mathrm{bpm}$ for 8 beats; pause setting to $4.5 \mathrm{~s}$; and AF setting to "AF only." Based on the implanting physician's preferences, other settings could be programmed. All devices were connected to the Medtronic CareLink network for remote monitoring.

\section{Clinical Follow-Up of ICM Group}

ICM patients were discharged on the day of implantation. Ten days after implantation, the patients were seen at the outpatient clinic to check the implantation site and to interrogate the ICM. Afterwards, the patients were seen regularly at the outpatient clinic according to routine patient care. ICM checkups were performed at the outpatient clinic every 6 months or earlier when necessary based on symptoms or transmitted episodes. Remote monitoring was performed on a daily basis during weekdays. All patient-activated episodes and automatically detected episodes were classified. In case of an inappropriate automatically detected episode, the cause of inappropriate detection was specified, if possible. Multiple actionable events could occur in 1 patient.

\section{Study Endpoints}

The primary endpoint of the study was the occurrence of an actionable event which was defined as an arrhythmic event resulting in any change in patient management (e.g., start or increase of medication, implantation of pacemaker or ICD, and catheter ablation). The secondary endpoint was the occurrence of any VT (at least 3 beats), irrespective if this resulted in an actionable event or not. A regular wide complex tachycardia was considered a VT if there was a sudden onset and a change in the QRS morphology in comparison to the baseline rhythm. An irregular wide complex tachycardia was considered a VT if there was a sudden onset and a polymorphic QRS morphology. A regular wide or narrow complex tachycardia was considered a supraventricular tachycardia (SVT) if there was a sudden onset and no change in QRS morphology. In case of doubt, a second electrophysiologist was consulted for the final diagnosis. For both endpoints, the cumulative event rate was determined at 30 months considering the estimated battery lifetime of the ICM.

\section{Statistical Analysis}

Continuous data are presented as mean \pm SD or as median with interquartile range (IQR) (25th and 75th percentiles), as appropriate. Categorical variables are presented by frequencies and percentages. Differences of continuous variables between groups were analyzed with unpaired Student's $t$ test or the Kruskal-Wallis test, as appropriate. Differences between categorical variables were evaluated using the $\chi^{2}$ test. Cumulative event rates were estimated with the Kaplan-Meier method, and differences were compared by log-rank test. Statistical analyses were performed using SPSS version 25 (IBM Corp., Somers, NY, USA). 


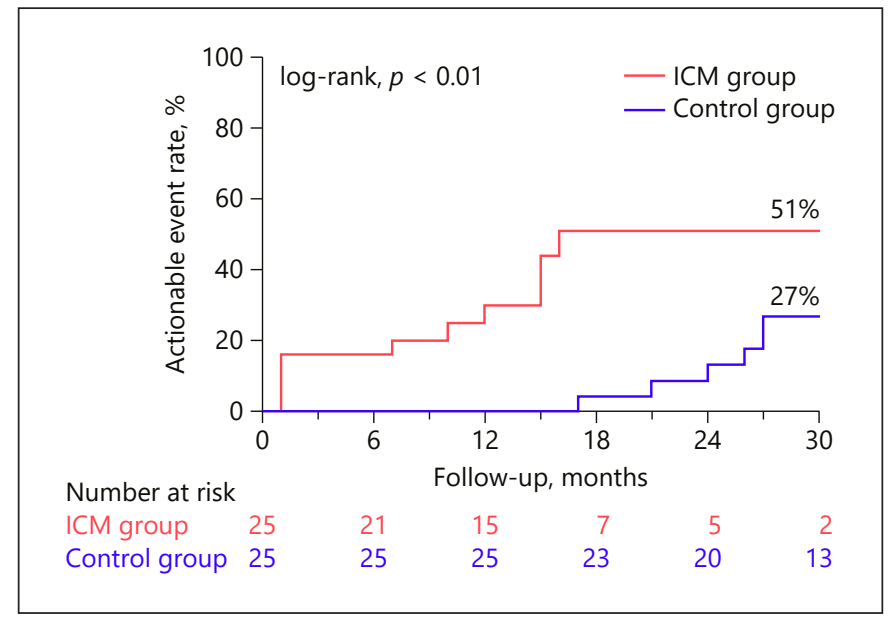

Fig. 1. Cumulative event rate for actionable events. ICM, insertable cardiac monitor.

\section{Results}

A total of $25 \mathrm{HCM}$ patients received an ICM between 2014 and 2019. We identified 25 matched controls with HCM who were seen at the outpatient clinic in the same study period. Baseline characteristics of the study population are presented in Table 1. The ICM group more often had a history of syncope ( $32 \%$ vs. $4 \%, p=0.01)$. Other baseline characteristics, including a history of nonsustained VT (NSVT), were similar between groups.

\section{ICM-Detected Arrhythmias and Holter Follow-Up}

During a mean follow-up of $17 \pm 10$ months with the ICM, a total of 1,015 episodes were transmitted to the CareLink network system. There were 270 (27\%) patientactivated episodes and 745 (73\%) automatically detected episodes. The majority of patient-activated episodes (93\%) comprised sinus rhythm with or without ectopy. In the control group, 48 Holter recordings were performed during follow-up. The median number of Holter recordings per patient was 2 (IQR, 1-3). The median interval between Holter recordings was 12 (IQR, 5-23) months.

\section{Primary Endpoint}

The cumulative event rate for an actionable event was higher in the ICM group (51 vs. $27 \%$ at 30 months, logrank $p$ value $<0.01$ ) (Fig. 1). In the ICM group, the following actionable events occurred: antiarrhythmic drug therapy (or change in dose) for documented arrhythmias $(n=6,24 \%)$, start of non-vitamin $\mathrm{K}$ antagonist oral anticoagulation for documented AF $(n=3,12 \%)$, electrophysiology study for symptomatic SVT $(n=2,8 \%)$, im-
Table 1. Clinical baseline characteristics

\begin{tabular}{|c|c|c|c|}
\hline Characteristic & $\begin{array}{l}\text { ICM } \\
\text { group } \\
(n=25)\end{array}$ & $\begin{array}{l}\text { Control } \\
\text { group } \\
(n=25)\end{array}$ & $\begin{array}{l}p \\
\text { value }\end{array}$ \\
\hline Age, years & $51 \pm 16$ & $52 \pm 16$ & 0.94 \\
\hline Sex, male & $18(72 \%)$ & $18(72 \%)$ & 1.00 \\
\hline NYHA functional class $\geq \mathrm{II}$ & $7(28 \%)$ & $7(28 \%)$ & 1.00 \\
\hline History of myectomy & $2(8 \%)$ & $3(12 \%)$ & 0.64 \\
\hline \multicolumn{4}{|l|}{ Left ventricular systolic function } \\
\hline Normal $(\mathrm{EF} \geq 50 \%)$ & $25(100 \%)$ & $24(96 \%)$ & 1.00 \\
\hline Mildly impaired (EF 45-49\%) & 0 & $1(4 \%)$ & \\
\hline Genetic testing & $21(84 \%)$ & $23(92 \%)$ & 0.67 \\
\hline Pathogenic mutation & $12(48 \%)$ & $14(56 \%)$ & 0.57 \\
\hline MYBPC3 & $8(32 \%)$ & $11(44 \%)$ & 0.38 \\
\hline MYH7 & $3(12 \%)$ & $1(4 \%)$ & 0.30 \\
\hline TPM1 & $1(4 \%)$ & 0 & 1.00 \\
\hline TNNI3 & 0 & $2(8 \%)$ & 0.49 \\
\hline History of NSVT & $13(52 \%)$ & $8(32 \%)$ & 0.15 \\
\hline History of unexplained syncope & $8(32 \%)$ & $1(4 \%)$ & 0.01 \\
\hline Peak LVOT gradient & $6(5-17)$ & $12(6-82)$ & 0.19 \\
\hline Family history of SCD & $3(12 \%)$ & $3(12 \%)$ & 1.00 \\
\hline Left atrial size & $43 \pm 9$ & $45 \pm 7$ & 0.23 \\
\hline \multicolumn{4}{|l|}{ Maximum left ventricular wall } \\
\hline thickness & $18 \pm 5$ & $18 \pm 5$ & 0.49 \\
\hline HCM Risk-SCD score & $3.41 \pm 1.31$ & $3.31 \pm 1.43$ & 0.79 \\
\hline$<4 \%$ & $13(52 \%)$ & $13(52 \%)$ & 1.00 \\
\hline$\geq 4$ to $\leq 6 \%$ & $12(48 \%)$ & $12(48 \%)$ & 1.00 \\
\hline Electrocardiography & $25(100 \%)$ & $25(100 \%)$ & 1.00 \\
\hline Sinus rhythm & $24(96 \%)$ & $25(100 \%)$ & 1.00 \\
\hline $\mathrm{AF}$ & $1(4 \%)$ & 0 & 1.00 \\
\hline PR interval, if sinus rhythm & $164 \pm 25$ & $182 \pm 25$ & 0.85 \\
\hline QRS duration, ms & $105 \pm 18$ & $102 \pm 28$ & 0.56 \\
\hline QTc duration, ms & $426 \pm 25$ & $416 \pm 28$ & 0.61 \\
\hline Holter monitoring & $25(100 \%)$ & $25(100 \%)$ & 1.00 \\
\hline$<1 \%$ PVCs & $23(92 \%)$ & $25(100 \%)$ & 0.49 \\
\hline $1-10 \%$ PVCs & $2(8 \%)$ & 0 & 0.49 \\
\hline NSVT & $11(44 \%)$ & $6(24 \%)$ & 0.14 \\
\hline SVT & $7(28 \%)$ & $12(48 \%)$ & 0.15 \\
\hline $\mathrm{AF}$ & $2(8 \%)$ & 0 & 0.49 \\
\hline Cardiac medication & $16(64 \%)$ & $19(76 \%)$ & 0.35 \\
\hline Beta blocker & $12(48 \%)$ & $14(56 \%)$ & 0.57 \\
\hline Loop diuretics & $5(20 \%)$ & 0 & 0.05 \\
\hline ACE-inhibitor/ARB & $6(24 \%)$ & $3(12 \%)$ & 0.27 \\
\hline Oral anticoagulants & $5(20 \%)$ & $5(20 \%)$ & 1.00 \\
\hline Calcium channel blocker & $3(16 \%)$ & $4(16 \%)$ & 0.68 \\
\hline Amiodarone/sotalol & $2(8 \%)$ & $2(8 \%)$ & 1.00 \\
\hline
\end{tabular}

Data are presented as $n(\%)$, mean $\pm \mathrm{SD}$, or median with IQR. ACE, angiotensin converting enzyme; $\mathrm{ARB}$, angiotensin receptor blocker; $\mathrm{CAD}$, coronary artery disease; EF, ejection fraction; HCM, hypertrophic cardiomyopathy; LVOT, left ventricular outflow tract; NSVT, nonsustained ventricular tachycardia; NYHA, New York Heart Association; PVC, premature ventricular complex; SCD, sudden cardiac death; IQR, interquartile range; SVT, supraventricular tachycardia; AF, atrial fibrillation.

plantation of ICD for primary prevention $(n=2,8 \%)$, pacemaker implantation for sinus node dysfunction $(n=$ $1,4 \%)$, and external electrical cardioversion for AF ( $n=$ $1,4 \%)$. In the control group, the following actionable events occurred: antiarrhythmic drug therapy (or change 


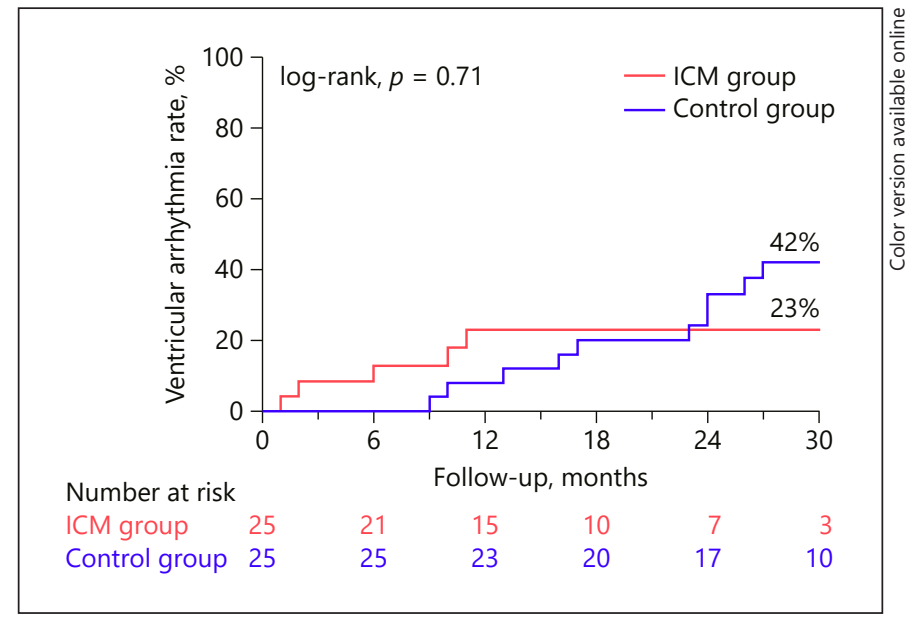

Fig. 2. Cumulative event rate for ventricular arrhythmias. ICM, insertable cardiac monitor.

in dose) for documented arrhythmias ( $n=6,24 \%)$, implantation of ICD for primary prevention $(n=2,8 \%)$, and electrophysiology study for symptomatic SVT $(n=1$, $4 \%)$. De novo AF only occurred in the ICM group. Of the 3 patients with de novo AF, only 1 patient who experienced symptoms required an electrical cardioversion for persistent AF.

Overall, 4 patients received an ICD for primary prevention ( 2 in each group). A patient in the ICM group had an ICM-detected NSVT (7 beats, $171 \mathrm{bpm}$, patientactivated) which increased his HCM Risk-SCD score from $3.6 \%$ to $8.0 \%$. He received a prophylactic ICD 15 months after his ICM implantation. The other patient in the ICM group had a history of NSVT, HCM RiskSCD score of $4.44 \%$, and patchy LGE anterior wall and interventricular septum on his CMR. He experienced an episode of fast monomorphic NSVT ( 32 beats, $200 \mathrm{bpm}$, automatically detected) 12 months post-ICM implantation. This did not increase his HCM Risk-SCD score, but based on his clinical profile and the malignant character of the VT, the patient received an ICD. In the control group, a patient received a prophylactic ICD after a Holter-detected monomorphic VT episode (3 beats, 135 bpm) increased his HCM Risk-SCD score from $4.4 \%$ to $9.6 \%$ at 27 months after initial risk evaluation. The second patient in the control group who received a prophylactic ICD had an HCM Risk-SCD score of $4.2 \%$ and had a history of NSVT. The combination of Holterdetected recurrent VT and recurrent near syncope was the indication for an ICD 26 months after initial risk evaluation.

\section{Secondary Endpoint}

The cumulative event rate for VT was $23 \%$ in the ICM group and $42 \%$ in the control group at 30 months (logrank $p$ value $=0.71$ ) (Fig. 2). Most VT episodes (4 of 5, $80 \%)$ in the ICM group were patient-activated episodes, and thus, were detected while patients experienced symptoms. One patient of the ICM group had a VT episode which was automatically detected (32 beats, $200 \mathrm{bpm}$ ). The characteristics of documented VT episodes were similar between groups with regard to the median number of documented beats (5 [IQR, 5-7] vs. 6 [IQR, 4-11], for the ICM group and the control group, respectively, $p=1.00)$ and median rate (150 bpm [IQR, 145-155 bpm] vs. $136 \mathrm{bpm}$ [IQR, 125-168 bpm], for the ICM group and the control group, respectively, $p=0.21$ ).

\section{Discussion}

The present study is the first study comparing the value of an ICM to conventional Holter monitoring in HCM patients with a low or intermediate HCM Risk-SCD score. The main finding is that actionable arrhythmic events occurred more frequently in the ICM group in comparison to the Holter group. In contrast, the cumulative rate of detected VT was similar between both groups.

It is well-known that prolonged arrhythmia monitoring increases the yield of arrhythmia detection. The indications for an ICM has expanded over the years, and its use is currently not only limited to patients with recurrent unexplained syncope $[1,8]$. Other important indications include the detection of subclinical AF, risk stratification in patients with inheritable heart disease by the detection of VT, and establishing a symptom-rhythm correlation in symptomatic patients [9]. In patients with $\mathrm{HCM}$ who are at low risk for SCD according to the HCM Risk-SCD score, the current ESC guidelines recommends that an ICM should be considered in patients with recurrent unexplained syncope and may be considered in those with unexplained palpitations $[1,2]$. However, limited data exist on the clinical impact of ICMs in HCM patients, and most studies comprised $<10$ patients [3-5].

The present study is the first to provide insight into the incremental value of an ICM in patients with HCM. The rate of actionable arrhythmic events was higher in the ICM group in comparison to a matched group who had intermittent Holter monitoring. Interestingly, de novo AF requiring oral anticoagulation only occurred in the ICM group. It is known that AF occurs in approximately $20 \%$ of the patients with HCM and is associated with im- 
paired quality of life, thromboembolism, and mortality [10-15]. To prevent thromboembolic complications, the guidelines recommend the use of lifelong oral anticoagulation, irrespective of the CHADS-VASc score, when AF occurs in patients with HCM [2]. Several studies in HCM patients with a cardiac implantable electronic device have demonstrated a high incidence of clinically silent AF episodes which may have important implications $[13,16]$. In our study, 3 patients (12\%) were started on oral anticoagulation after the detection of de novo AF detected by the ICM. Thus, an ICM may play a role in the detection of subclinical AF in this specific population.

SCD is the most feared consequence of HCM, which has led to meticulous efforts to identify those patients who may benefit from a prophylactic ICD. Since 2014, the HCM Risk-SCD model provides guidance to physicians to identify patients deemed to be at high risk for SCD, and thus eligible for a prophylactic ICD $[17,18]$. In clinical practice, however, we are confronted with patients with a low or intermediate risk who have additional risk factors which are not incorporated in the HCM Risk-SCD model, such as extensive LGE on CMR [19], LV apical aneurysms, multiple pathogenic sarcomere protein variants, and LV dysfunction. The presence of NSVT is an important risk factor, especially in those patients younger than 30 years of age $[20,21]$. In the American guidelines, there is class IIa indication for an ICD in patients with NSVT who have additional SCD modifiers (i.e., age $<30$ years, LGE on CMR, LVOT obstruction, LV aneurysm, and syncope $>5$ years ago) [6]. Considering the clinical relevance of documenting VT in this population, routine ambulatory Holter monitoring is recommended $[2,6,7]$.

We expected that continuous monitoring with an ICM would improve VT detection. Interestingly, in our study, the diagnostic yield for detecting VT was similar between the ICM and Holter groups. This apparent paradox can be partly explained by the ICM settings. Only longer and faster runs of VT ( $\geq 16$ beats at a rate of $>176 \mathrm{bpm}$ ) or symptomatic VT (patient-activated episodes) will be detected by the ICM, while with Holter monitoring, a VT of $\geq 3$ beats at a rate of $>120 \mathrm{bpm}$ will suffice. The Reveal LINQ can be programmed to detect a tachycardia of 5 beats at a rate of $>120 \mathrm{bpm}$. However, this sensitive programming setting will result in a suboptimal signal-to-noise ratio, as many tachycardia episodes will be due to sinus tachycardia.

There is some inconsistency in literature with regard to the prognostic significance of specific characteristics of documented VT. Studies in unselected HCM cohorts have shown no association between characteristics of the detected VT on ambulatory Holter monitoring and the occur- rence of SCD [21]. However, several HCM cohorts with ICDs (higher-risk cohorts) demonstrated that longer-lasting and faster VTs were more predictive of the occurrence of appropriate ICD therapy [22-24]. For example, in 160 HCM patients with ICDs, Wang et al. [24] reported the independent association of fast $(>200 \mathrm{bpm})$, long $(>7$ beats), and repetitive runs of VT with the occurrence of ICD therapy, whereas this association was not shown for slower, shorter, and single run of VT [24]. These data are important and support the use of ICMs as these devices are able to capture the more predictive longer and faster VT.

Finally, there are some factors that need to be considered when using ICMs in this patient population, including device costs, data overload, the clinical relevance of detected arrhythmias, and medical overuse. A dedicated telemonitoring staff is a requirement before providing such a service to patients.

\section{Study Limitations}

Although we used a matched control group, the present study is a nonrandomized study and selection bias is possible. The control group had a lower proportion of patients with a history of syncope. It is important to stress that the ICM population was not a general HCM population, but a selected cohort of HCM patients with symptoms or additional risk factors for SCD. Finally, the classification of wide complex tachycardia as either VT or SVT can be challenging considering that only a single surface electrogram is available. To reduce the risk of misclassification, difficult electrograms were reevaluated by an electrophysiologist.

\section{Conclusions}

In patients with HCM, the use of an ICM resulted in more actionable arrhythmic events if compared to intermittent Holter monitoring. Interestingly, de novo AF was only detected in the ICM group. The diagnostic yield of detecting VT appeared similar between both rhythm detection strategies, which may be explained by the ICM not detecting short runs of VT.

\section{Statement of Ethics}

The Institutional Review Board of the Erasmus MC reviewed the study (MEC2017052), and this study was not subjected to the Dutch Medical Research Involving Human Subjects Act. This study was carried out according to the ethical principles for medical research involving human subjects established by Declaration of Helsinki, protecting the privacy of all the participants and the confidentiality of their personal information. 


\section{Conflict of Interest Statement}

Dr. Yap has received a research grant from Medtronic.

\section{Funding Sources}

The authors did not receive any funding.

\section{Author Contributions}

All authors fulfil the ICMJE criteria for authorship. S.C.Y., J.W.R.H., and M.M. designed the study. R.S., R.H., and A.A. were responsible for acquisition and analysis of data and drafting the manuscript. D.A.M.J.T., A.F.L.S., and T.S.T. were responsible for interpretation of data. S.C.Y., T.S.T., J.W.R.H., D.A.M.J.T., M.M., and A.F.L.S. critically revised the manuscript. All authors have read and approved the final version of the manuscript and take responsibility for the work.

\section{References}

1 Brignole M, Moya A, de Lange FJ, Deharo JC, Elliott PM, Fanciulli A, et al. 2018 ESC guidelines for the diagnosis and management of syncope. Eur Heart J. 2018;39(21):1883-948.

2 Authors/Task Force members, Elliott PM, Anastasakis A, Borger MA, Borggrefe M, Cecchi F, Charron P, et al. ESC guidelines on diagnosis and management of hypertrophic cardiomyopathy: the task force for the diagnosis and management of hypertrophic cardiomyopathy of the European Society of Cardiology (ESC). Eur Heart J. 2014;35:2733-79.

3 Sakhi R, Theuns DAMJ, Bhagwandien RE, Michels M, Schinkel AFL, Szili-Torok T, et al. Value of implantable loop recorders in patients with structural or electrical heart disease. J Interv Card Electrophysiol. 2018;52(2): 203-8.

4 Pezawas T, Stix G, Kastner J, Schneider B, Wolzt $\mathrm{M}$, Schmidinger $\mathrm{H}$. Implantable loop recorder in unexplained syncope: classification, mechanism, transient loss of consciousness and role of major depressive disorder in patients with and without structural heart disease. Heart. 2008;94(4):e17.

5 Magnusson P, Mörner S. EvaLuation using cardiac insertable devices and telephone in hypertrophic cardiomyopathy (ELUCIDATE HCM)-rationale and design: a prospective observational study on incidence of arrhythmias in Sweden. BMJ Open. 2017;7(12):e019541.

6 Al-Khatib SM, Stevenson WG, Ackerman MJ, Bryant WJ, Callans DJ, Curtis AB, et al. AHA/ ACC/HRS guideline for management of patients with ventricular arrhythmias and the prevention of sudden cardiac death: a report of the American college of cardiology/American heart association task force on clinical practice guidelines and the heart rhythm society. J Am Coll Cardiol. 2018 Oct 2;72(14): e91-e220.

7 Priori SG, Blomstrom-Lundqvist C, Mazzanti A, Blom N, Borggrefe M, Camm J, et al. ESC guidelines for the management of patients with ventricular arrhythmias and the prevention of sudden cardiac death: the task force for the management of Patients with ventricular arrhythmias and the prevention of sudden cardiac death of the European society of cardiology (ESC). Endorsed by: association for European paediatric and congenital cardiology (AEPC). Eur Heart J. 2015;36:2793-867.

8 Brignole M, Brignole M, Vardas P, Hoffman E, Huikuri H, Moya A, et al. Indications for the use of diagnostic implantable and external ECG loop recorders. Europace. 2009;11(5): 671-87.

9 Sakhi R, Theuns DAMJ, Szili-Torok T, Yap $S C$. Insertable cardiac monitors: current indications and devices. Expert Rev Med Devices. 2019;16(1):45-55.

10 Olivotto I, Cecchi F, Casey SA, Dolara A, Traverse $\mathrm{JH}$, Maron BJ. Impact of atrial fibrillation on the clinical course of hypertrophic cardiomyopathy. Circulation. 2001;104(21): 2517-24.

11 Ho CY, Day SM, Ashley EA, Michels M, Pereira AC, Jacoby D, et al. Genotype and lifetime burden of disease in hypertrophic cardiomyopathy: insights from the sarcomeric human cardiomyopathy registry (SHaRe). Circulation. 2018;138(14):1387-98.

12 Healey JS, Connolly SJ, Gold MR, Israel CW, Van Gelder IC, Capucci A, et al. Subclinical atrial fibrillation and the risk of stroke. $\mathrm{N}$ Engl J Med. 2012;366(2):120-9.

13 van Velzen HG, Theuns DA, Yap SC, Michels $\mathrm{M}$, Schinkel AF. Incidence of device-detected atrial fibrillation and long-term outcomes in patients with hypertrophic cardiomyopathy. Am J Cardiol. 2017;119(1):100-5.

14 Guttmann OP, Rahman MS, O’Mahony C, Anastasakis A, Elliott PM. Atrial fibrillation and thromboembolism in patients with hypertrophic cardiomyopathy: systematic review. Heart. 2014;100(6):465-72.

15 Rowin EJ, Hausvater A, Link MS, Abt P, Gionfriddo W, Wang W, et al. Clinical profile and consequences of atrial fibrillation in hypertrophic cardiomyopathy. Circulation. 2017;136:2420-36.

16 Rowin EJ, Orfanos A, Estes NAM, Wang W, Link MS, Maron MS, et al. Occurrence and natural history of clinically silent episodes of atrial fibrillation in hypertrophic cardiomyopathy. Am J Cardiol. 2017;119(11):1862-5.
17 O’Mahony C, Akhtar MM, Anastasiou Z, Guttmann OP, Vriesendorp PA, Michels M, et al. Effectiveness of the 2014 European society of cardiology guideline on sudden cardiac death in hypertrophic cardiomyopathy: a systematic review and meta-analysis. Heart. 2019;105(8):623-31.

18 O'Mahony C, Jichi F, Pavlou M, Monserrat L, Anastasakis A, Rapezzi C, et al. A novel clinical risk prediction model for sudden cardiac death in hypertrophic cardiomyopathy (HCM risk-SCD). Eur Heart J. 2014;35(30): 2010-20.

19 Briasoulis A, Mallikethi-Reddy S, Palla M, Alesh I, Afonso L. Myocardial fibrosis on cardiac magnetic resonance and cardiac outcomes in hypertrophic cardiomyopathy: a meta-analysis. Heart. 2015;101(17):1406-11.

20 Christiaans I, van Engelen K, van Langen IM, Birnie E, Bonsel GJ, Elliott PM, et al. Risk stratification for sudden cardiac death in hypertrophic cardiomyopathy: systematic review of clinical risk markers. Europace. 2010; 12(3):313-21.

21 Monserrat L, Elliott PM, Gimeno JR, Sharma S, Penas-Lado M, McKenna WJ. Non-sustained ventricular tachycardia in hypertrophic cardiomyopathy: an independent marker of sudden death risk in young patients. J Am Coll Cardiol. 2003;42(5):873-9.

22 Francia P, Santini D, Musumeci B, Semprini L, Adduci C, Pagannone E, et al. Clinical impact of nonsustained ventricular tachycardia recorded by the implantable cardioverter-defibrillator in patients with hypertrophic cardiomyopathy. J Cardiovasc Electrophysiol. 2014;25(11):1180-7.

23 Viswanathan K, Suszko AM, Das M, Jackson N, Gollob M, Cameron D, et al. Rapid devicedetected nonsustained ventricular tachycardia in the risk stratification of hypertrophic cardiomyopathy. Pacing Clin Electrophysiol. 2016;39(7):642-51.

24 Wang W, Lian Z, Rowin EJ, Maron BJ, Maron MS, Link MS. Prognostic implications of nonsustained ventricular tachycardia in high-risk patients with hypertrophic cardiomyopathy. Circ Arrhythm Electrophysiol. 2017;10(3). 